\title{
Protective effect of superhydrophobic coatings on carbon steel in different environments
}

\author{
V.I. Vigdorovich ${ }^{+},{ }^{1}$ L.E. Tsygankova, ${ }^{1,2}{ }^{-} *$ M.N. Uryadnikova, ${ }^{2}$ \\ K.A. Emel'yanenko, ${ }^{3}$ E.V. Chulkova ${ }^{3}$ and A.A. Uryadnikov ${ }^{1,2}$
}

${ }^{1}$ All-Russian Scientific Research Institute of Use of Machinery and Oil Products, Novo-Rubezhnyi per., 28, Tambov, 392022, Russian Federation

${ }^{2}$ Derzhavin State University, ul. Internatsyonalnaya, 33, Tambov, 392000, Russian Federation

${ }^{3}$ A.N. Frumkin Institute of Physical Chemistry and Electrochemistry, Russian Academy of Sciences, Leninsky pr. 31, 119071 Moscow, Russian Federation *E-mail: vits21@mail.ru

\begin{abstract}
The features of the protective efficiency of nanocomposite superhydrophobic coatings of 4 types on carbon steel are considered. The first type of coating was obtained by texturing the St3 steel surface using nanosecond IR laser radiation with subsequent chemisorption of methoxy 3 $[(2,2,3,3,4,4,5,5,6,6,7,7,8,8,8$-pentadecafluorooctyl)oxy]propyl $\}$ silane from solution in $n$ decane (MAF). To obtain a type II coating, a nano-sized composite layer consisting of Aerosil nanoparticle aggregates coated with the same hydrophobic agent was additionally applied on the type I coating. To obtain a type III coating, the St3 working surface was textured with a nanosecond IR laser, followed by chemisorption of a hydrophobizing agent, $\mathrm{CF}_{3}\left(\mathrm{CF}_{2}\right)_{6}\left(\mathrm{CH}_{2}\right) \mathrm{O}-$ $\left(\mathrm{CH}_{2}\right)_{2} \mathrm{C}\left(\mathrm{OCH}_{3}\right)_{3}$, from the vapor phase. Type IV coatings were obtained by texturing the steel surface with a nanosecond IR laser followed by treatment with ultraviolet radiation in the presence of ozone plasma. Further, for hydrophobization, the samples were kept in a closed container containing saturated vapors of the methoxy\{3-[(2,2,3,3,4,4,5,5,6,6,7,7,8,8,8pentadecafluorooctyl)oxy]propyl $\}$ silane hydrophobizing agent. Then heat treatment was carried out at $150^{\circ}$. The contact angle on the obtained samples was $168-172^{\circ}$. Gravimetric corrosion tests and polarization measurements, as well as measurements of the contact angle on the obtained superhydrophobic samples over time were conducted. Studies have been carried out in neutral chloride medium, NACE medium $+400 \mathrm{mg} / \mathrm{L} \mathrm{H}_{2} \mathrm{~S}$ and under conditions of $100 \%$ humidity. In the latter case, superhydrophobic coatings of types I and II did not undergo changes within 112 days. In a neutral chloride medium, their protective effect is close to $96 \%$. An environment with $\mathrm{H}_{2} \mathrm{~S}$ causes the fragility of the anti-corrosion properties of the super-hydrophobic coating. The use of ultraviolet irradiation treatment in the presence of ozone plasma during the formation of superhydrophobic coatings contributes to an increase in their anticorrosion properties.
\end{abstract}

Received: August 9, 2021. Published: August 25, 2021

doi: $\underline{10.17675 / 2305-6894-2021-10-3-19}$

Keywords: steel, superhydrophobic coating, contact angle, corrosion rate, protective effect, sodium chloride, hydrogen sulfide. 


\section{Introduction}

In the past decade, studies devoted to the anticorrosive efficiency of hydrophobic (surface contact angle $\left.\theta \geq 120^{\circ}\right)$ and superhydrophobic coatings $\left(\theta=150-170^{\circ}\right)$ on metals have been intensively developed [1-4]. The main mechanisms of the protective action of such coatings under conditions of electrochemical corrosion are associated with:

- preventing the supply of water molecules to the metal surface, the presence of which is necessary for partial electrode reactions to occur [5];

- blocking of adsorption-active surface centers with molecules of a hydrophobic agent [6];

- electrostatic repulsion of anions due to negative charging of the "hydrophobic coating/electrolyte solution" interface [6].

Usually, the protective effectiveness of such coatings is studied in a neutral chloride environment $[4,6]$. Electrochemical corrosion rate is determined based on polarization curves or linear polarization resistance method. Direct corrosion tests are usually not performed. The possibility of measuring polarization curves on metals with a superhydrophobic coating indicates that contact of a metal surface with an electrolyte solution is not completely excluded. A number of studies claim that the total proportion of the wetted area for superhydrophobic surfaces, where a rough metal coated with a layer of a hydrophobic agent is in direct contact with a liquid solution, can reach $10 \%$ [2, 7]. At the same time, hydrophobic and superhydrophobic surface films are not continuous. An electric double layer at the metal/solution interface that occurs along the wetted area is responsible for the occurrence of electrochemical corrosion.

The aim of this work is to compare the protective effectiveness of superhydrophobic coatings, determined by the method of polarization curves and direct corrosion tests in environments that differ in their activity.

\section{Experimental}

Superhydrophobic coatings (SHPC) of 4 types were studied, which were applied to the surface of steel electrodes in the Laboratory of Surface forces at the Institute of Physical Chemistry and Electrochemistry, Russian Academy of Sciences. To obtain a type I coating, the surface of St3 steel was textured using nanosecond IR laser radiation with subsequent chemisorption of methoxy-\{3-[(2,2,3,3,4,4,5,5,6,6,7,7,8,8,8-pentadecafluorooctyl)oxy]propyl $\}$ silane from solution in $n$-decane (MAF). To obtain a type II coating, a nano-sized composite layer consisting of aggregates of Aerosil nanoparticles coated with the same hydrophobic agent was additionally applied to the type I coating. The wetting angle on the initial steel specimen was $85.3 \pm 2.7^{\circ}$, on superhydrophobic specimens of both types $168 \pm 1.5^{\circ}$.

To obtain a type III coating, the St3 working surface was textured with a nanosecond IR laser with the following laser processing parameters: pulse duration $50 \mathrm{~ns}$, pulse frequency $20 \mathrm{kHz}$, peak pulse power $0.95 \mathrm{~mJ}$, linear laser beam rate $50 \mathrm{~mm} / \mathrm{s}$, scanning 
density 150 lines per $\mathrm{mm}$. To impart superhydrophobic properties to the surface, the surface energy of the formed texture was lowered by chemisorption of the $\mathrm{CF}_{3}\left(\mathrm{CF}_{2}\right)_{6}\left(\mathrm{CH}_{2}\right) \mathrm{O}\left(\mathrm{CH}_{2}\right)_{2} \mathrm{C}\left(\mathrm{OCH}_{3}\right)_{3}$ hydrophobizing agent from the vapor phase, followed by drying of the resulting coating at $130^{\circ} \mathrm{C}$ for 1 hour. The wetting angle on the obtained superhydrophobic samples was $165 \pm 2^{\circ}$, and the rolling angle for droplets with a volume of $15 \mu \mathrm{L}$ was $3 \pm 1^{\circ}$. The samples under study (due to design features: the samples were pressed into a fluoroplastic frame with a visible area of the working surface close to $0.5 \mathrm{~cm}^{2}$ ) were not treated with ultraviolet irradiation in the presence of ozone plasma.

Type IV coatings were obtained on St 3 steel samples $10 \times 20 \times 3 \mathrm{~mm}$ in size. The working surface of St3 was textured with a nanosecond IR laser, followed by ultrasonic cleaning of the surface with an operating frequency of $35 \mathrm{kHz}$ and a sound generator power of $55 \mathrm{~W}$ to remove poorly fixed particles from the surface of the samples. For hydrophobization, the samples textured in the above mode of laser treatment and treated with ultraviolet radiation in the presence of ozone plasma were placed in a closed container containing saturated vapors of methoxy $\{3-[(2,2,3,3,4,4,5,5,6,6,7,7,8,8,8$-pentadecafluorooctyl)oxy]propyl $\}$ silane as a hydrophobizing agent. The container with the samples was placed in an oven, heated to a temperature of $95^{\circ} \mathrm{C}$ and kept at this temperature for 2 hours, and then cooled to room temperature. After removing from the container with the hydrophobizator, the samples were washed with industrial alcohol to remove physically adsorbed molecules, dried with filter paper and heat treated in an oven for 1 hour at a temperature of $150^{\circ} \mathrm{C}$. With this heat treatment, the adsorbed molecules cross-link with each other to form a two-dimensional polymeric hydrophobic layer. The wetting angle on the obtained superhydrophobic samples was $172.6 \pm 1.2^{\circ}$, the roll-off angle was $2.5 \pm 1^{\circ}$.

The studies were carried out on St 3 carbon steel with a chemical composition, mass \%: $\mathrm{C}-0.20 ; \mathrm{Mn} 0.50 ; \mathrm{Si}-0.15 ; \mathrm{P}-0.04 ; \mathrm{S}-0.05 ; \mathrm{Cr} 0.31 ; \mathrm{Ni}-0.23 ; \mathrm{Cu} 0.21 ; \mathrm{Fe}$ - rest.

The electrochemical and corrosion behavior of $\mathrm{St} 3$ carbon steel with a superhydrophobic coating was studied in $0.5 \mathrm{M} \mathrm{NaCl}$ solution and NACE medium $(5 \mathrm{~g} / \mathrm{L}$ $\left.\mathrm{NaCl}+0.25 \mathrm{~g} / \mathrm{L} \mathrm{CH}_{3} \mathrm{COOH}, \mathrm{pH}=3.6\right)+400 \mathrm{mg} / \mathrm{L} \mathrm{H}_{2} \mathrm{~S}$.

Gravimetric corrosion studies at $100 \%$ humidity were carried out at room temperature in hermetically sealed desiccators with a volume of 7 liters, in which $100 \%$ relative humidity was achieved by evaporation of distilled water from an container with a large water/air surface area placed inside the desiccator. The samples were suspended on nylon threads that did not swell in a humid atmosphere. For comparison, corrosion studies were performed on untreated samples and samples with superhydrophobic coatings.

A number of corrosion tests were carried out in NACE medium $+400 \mathrm{mg} / \mathrm{L} \mathrm{H}_{2} \mathrm{~S}$ solutions for 24 hours and 10 days on samples with and without a protective coating. In both cases, the samples were weighed before and after exposure. The corrosion rate was calculated from the weight loss of the samples during the experiment.

The protective effect of the superhydrophobic coating $(Z, \%)$ was calculated by the formula: 


$$
Z, \%=100\left(K_{0}-K_{\text {SHPC }}\right) / K_{0},
$$

where $K_{0}$ and $K_{\text {SHPC }}$ are the corrosion rates of uncoated and coated electrodes, respectively.

In some cases, after exposure of the samples, the contact angles were measured at 3-5 different points on the surface using a tensiometer (Easy Drop, KRUSS, Germany). A scanning electron microscope (JSM 6390, JEOL, Japan) was used to assess the condition of the coating before and after corrosion exposure.

Polarization potentiodynamic (potential scan rate of $0.66 \mathrm{mV} / \mathrm{s}$ ) curves were measured on electrodes without and with a protective coating according to the method described in [8].

The polarization curves were measured 15 minutes after the electrode was immersed in the solution and every day for 168 hours without removing from the solution. Corrosion currents $\left(i_{\text {cor }}\right)$ were calculated by extrapolating the Tafel sections of the polarization curves to the corrosion potential. The protective effect of the hydrophobic coating was calculated by the formula:

$$
Z, \%=100 \cdot\left(i_{\mathrm{cor}, 0}-i_{\mathrm{cor}, \mathrm{SHPC}}\right) / i_{\mathrm{cor}, 0}
$$

\section{Results and Discussion}

\section{Gravimetric corrosion test data}

\section{Results at $100 \%$ humidity}

Table 1 shows the results of testing steel samples at 100\% humidity from which it follows that for 112 days, electrodes with superhydrophobic coatings did not show signs of corrosion damage. For type 4 electrodes, in parallel with weighing, the contact angles on the surface were measured at regular intervals. The value of the contact angle, both after 14-day exposure and after 112 days, did not differ from the initial one (Figure 1).

Table 1. Corrosion rate $\left(K, \mathrm{~g} /\left(\mathrm{m}^{2} \mathrm{~h}\right)\right)$ of unprotected and superhydrophobic steel in an atmosphere of desiccators with $100 \%$ relative humidity.

\begin{tabular}{ccccc}
\hline \multirow{2}{*}{$\begin{array}{c}\text { Exposure } \\
\text { duration, days }\end{array}$} & \multicolumn{4}{c}{ Corrosion rate $\left(\boldsymbol{K}, \mathbf{g} /\left(\mathbf{m}^{2} \mathbf{h}\right)\right):$} \\
\cline { 2 - 5 } & Unprotected steel & Type I coating & Type II coating & Type IV coating \\
\hline 14 & 0.012 & 0 & 0 & 0 \\
28 & 0.026 & 0 & 0 & 0 \\
56 & 0.064 & 0 & 0 & 0 \\
112 & not rated & 0 & 0 & 0 \\
\hline
\end{tabular}



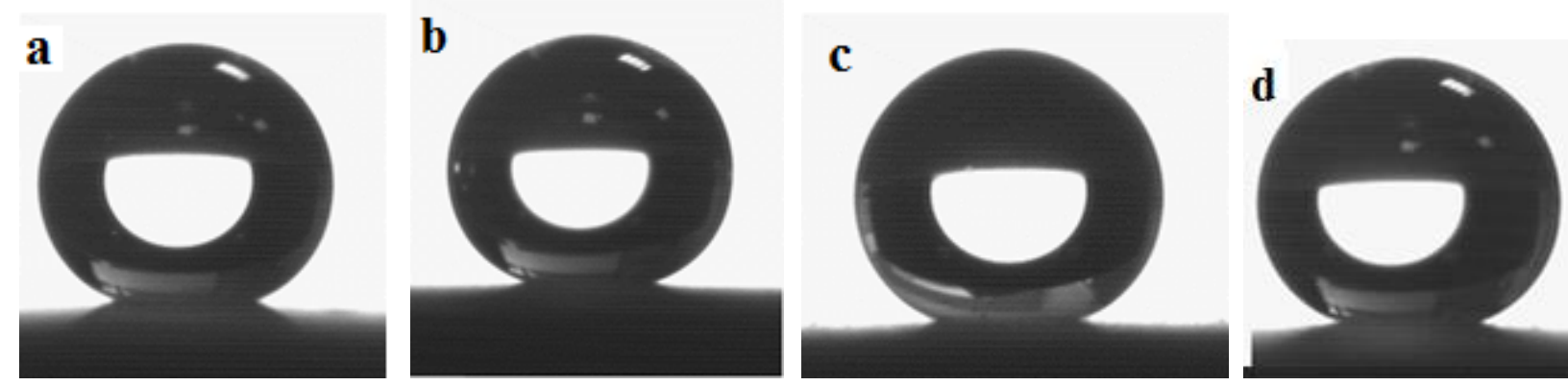

Figure 1. Images of water droplets on the surface of an electrode with a superhydrophobic coating after exposure at $100 \%$ humidity for: $\mathrm{a}-$ before the experiment, $\mathrm{b}-14$ days, $\mathrm{c}-56$ days, $\mathrm{d}-112$ days.

\section{Results in NACE medium $+400 \mathrm{mg} / \mathrm{L} \mathrm{H}_{2} \mathrm{~S}$}

Samples with coatings of the IV type were investigated. After 24 hours of exposure, as well as after 240 hours, the surface of the electrodes was covered with corrosion products of black color with a brown tint (Figure 2). After removing powdery corrosion products, washing the electrodes with water and drying in air for an hour, the contact angle was $63^{\circ}$ in the first case and $66^{\circ}$ in the second case.

SEM images of the electrode surface before the experiment and after exposure to the solution are shown in Figure 3.

After 24 hours of

exposure

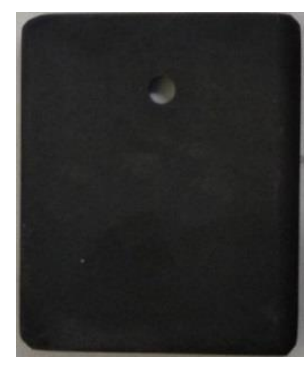

After 240 hours of exposure

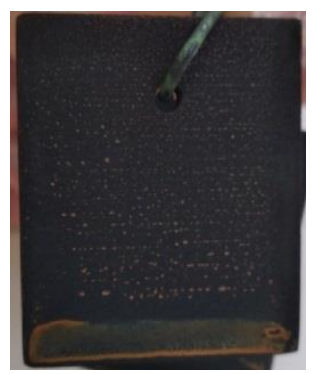

After removing powdery corrosion products

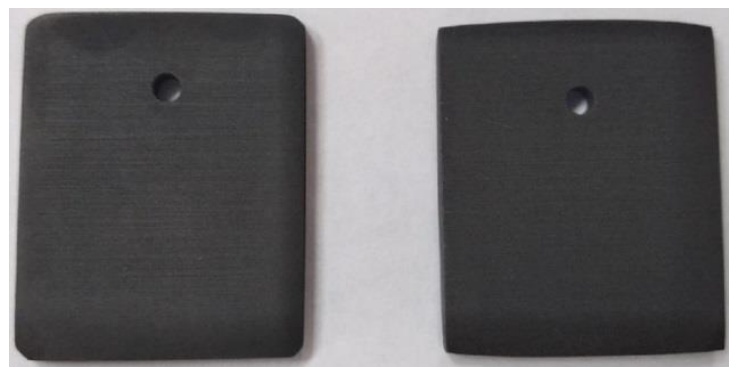

Figure 2. Appearance of samples after exposure to NACE medium $+400 \mathrm{mg} / \mathrm{L} \mathrm{H}_{2} \mathrm{~S}$ solution.

In Figure 3a, it can be seen that the texture of the coating surface before testing has the form of grooves evenly spaced from each other, on which, as well as in the intervals between them, rod-like and spherical aggregates of nanoparticles are visible. After exposure to the solution, the surface is smoothed, the grooves are only slightly visible (Figure 3b,c). It is possible that the smoothing is due to the formation of sulfide corrosion products on the surface, which are also the reason for the decrease in the contact angle to $<90^{\circ}$. But the protective effect of the coating persists, giving a $Z$ value of $86 \%$ after 24 hours and $65 \%$ after 240 hours of exposure (Table 2). 


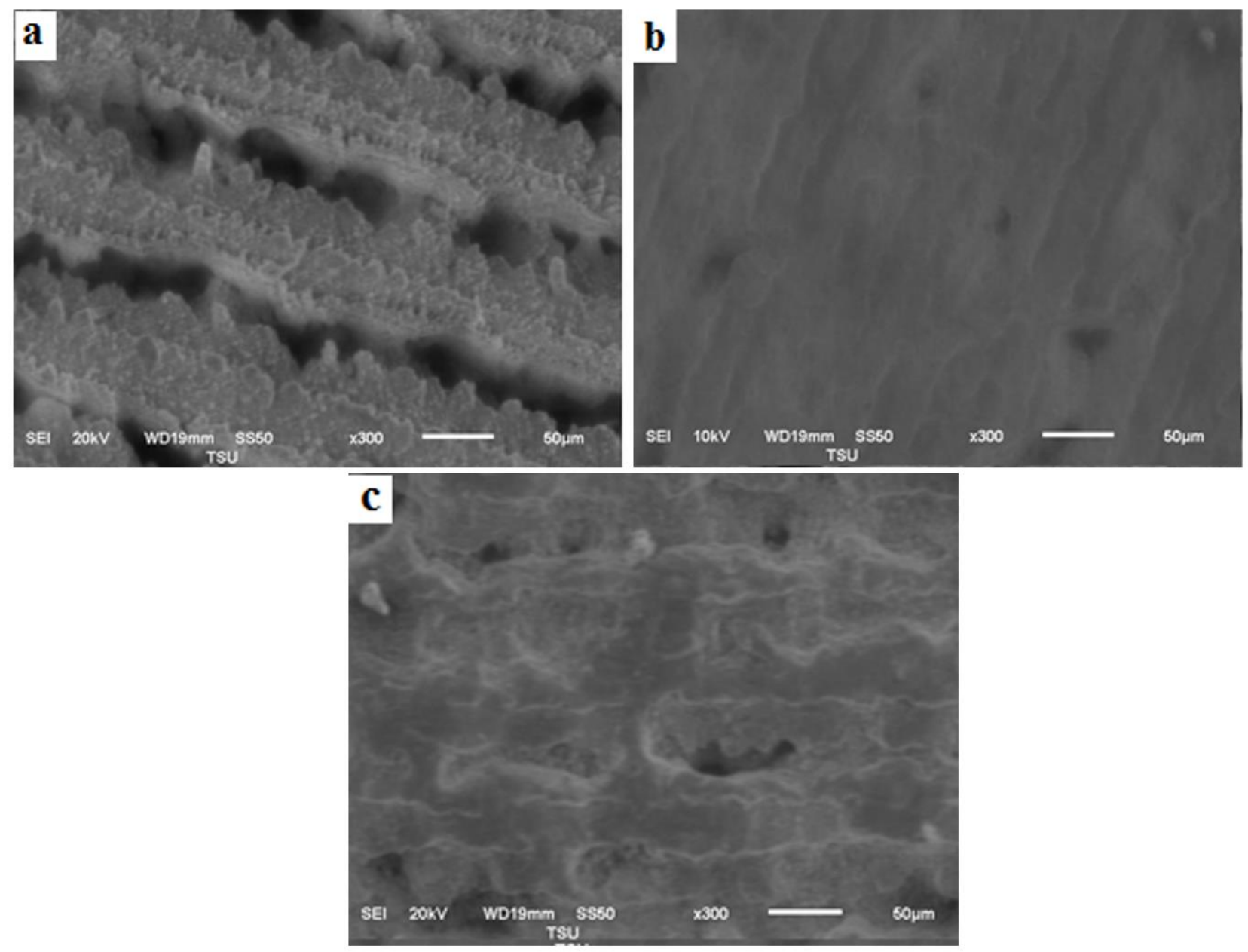

Figure 3. SEM images of the surface of steel with superhydrophobic type IV coating before experiment (a) and after exposure in NACE medium $+400 \mathrm{mg} / \mathrm{L} \mathrm{H}_{2} \mathrm{~S}$ for 24 hours (b) and 240 hours (c).

Table 2. Corrosion rate $K, \mathrm{~g} /\left(\mathrm{m}^{2} \mathrm{~h}\right)$ and protective effect $Z, \%$ of type IV superhydrophobic coating in NACE medium $+400 \mathrm{mg} / \mathrm{L} \mathrm{H}_{2} \mathrm{~S}$ solution, calculated from the data on sample weight loss during exposure.

\begin{tabular}{ccc}
\hline \multirow{2}{*}{ Sample } & \multicolumn{2}{c}{ Exposure time in solution, $\mathbf{h}$} \\
\cline { 2 - 3 } & $\mathbf{2 4}$ & $\mathbf{2 4 0}$ \\
\cline { 2 - 3 } & $\boldsymbol{K} / \boldsymbol{K}$ & $\boldsymbol{K} / \boldsymbol{Z}$ \\
\hline Uncoated steel & $0.522 /-$ & $0.201 /-$ \\
Coated steel & $0.072 / 86$ & $0.070 / 65$ \\
\hline
\end{tabular}

Table 2 shows that the corrosion rate of type IV coated electrodes practically did not change with an increase in the exposure time in the solution from 24 to 240 hours. At the same time, the corrosion rate of unprotected samples decreased 2.6 times due to the formation of a protective sulfide film of corrosion products on the surface, as is usually 
observed in hydrogen sulfide media [9]. Therefore, the protective effectiveness of the superhydrophobic coating formally decreased with an increase in the duration of the test. As follows from the literature [10], corrosion of hydrophobized samples can be associated with the hydrolysis of the $-\mathrm{O}-\mathrm{Si}-$ bond of the hydrophobizator molecule in acidic media and partial desorption of fluorooxysilane from the coating into the solution, which is in good agreement with the decrease in the contact angle. However, the fact that the corrosion rate of samples with a superhydrophobic coating is practically constant over time deserves attention and can be explained by the healing of active surface areas with sulfide corrosion products, the presence of which also contributes to a decrease in the contact angle to $<90^{\circ}$.

\section{Polarization measurements in NACE medium $+400 \mathrm{mg} / \mathrm{L} \mathrm{H}_{2} \mathrm{~S}$ solution}

At the initial stage of corrosion $(0.25 \mathrm{~h})$, electrodes without coating and with superhydrophobic coating of the III type have the same corrosion potentials (Figure 4). On the protected electrode, both electrode reactions are inhibited compared to the uncoated electrode. The protective effect of the coating $Z=65 \%$ was determined on the basis of the corrosion currents calculated by extrapolating the linear sections of the polarization curves to the corrosion potential. After a day, the $E_{\text {cor }}$ of the unprotected electrode shifts to the region of more negative potentials by $100 \mathrm{mV}$, while that of the protected electrode, by only $10 \mathrm{mV}$. The corrosion rates of both electrodes decreased, but that of the protected electrode decreased to a greater extent, so the protective effect of the coating increased to $85 \%$ (Table 3).

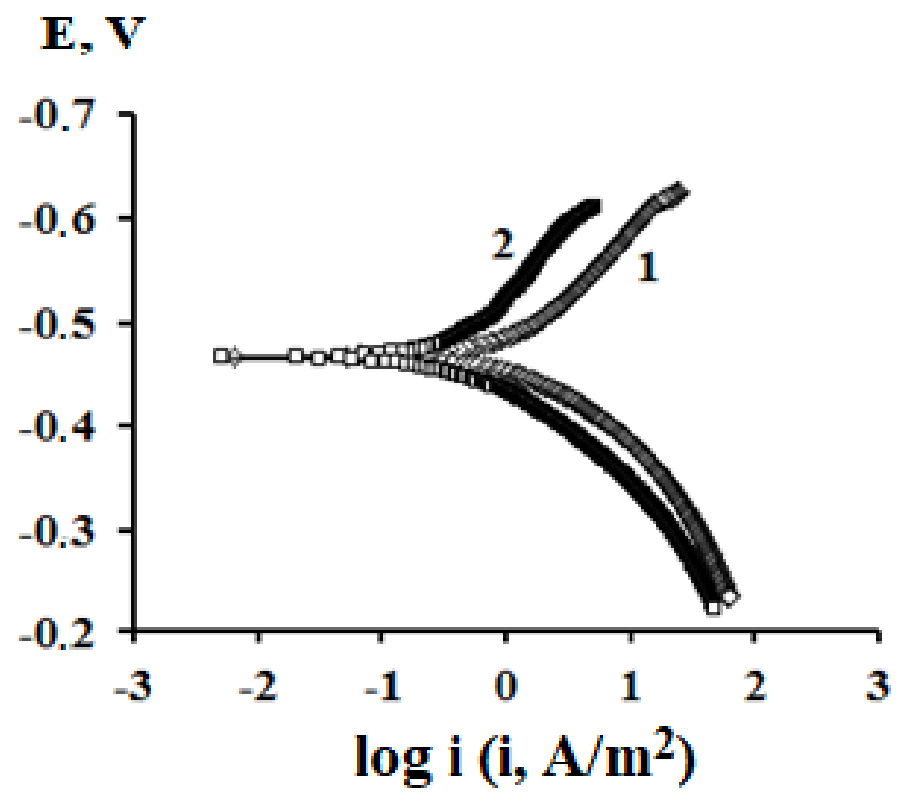

Figure 4. Polarization curves measured on a steel electrode without a coating (1) and with type III superhydrophobic coating (2) after exposure to NACE medium $+400 \mathrm{mg} / \mathrm{L} \mathrm{H}_{2} \mathrm{~S}$ solution for $15 \mathrm{~min}$. 
Table 3. Corrosion rate $\left(i_{\mathrm{cor}}, \mathrm{A} / \mathrm{m}^{2}\right)$, corrosion potential $\left(E_{\mathrm{cor}}, \mathrm{V}\right)$ and protective effect $(Z, \%)$ of type III superhydrophobic coating on a steel electrode compared to an unprotected electrode in NACE medium + $400 \mathrm{mg} / \mathrm{L} \mathrm{H}_{2} \mathrm{~S}$ environment.

\begin{tabular}{cccccc}
\hline \multirow{2}{*}{$\begin{array}{c}\text { Time of } \\
\text { exposure, } \mathbf{h}\end{array}$} & \multicolumn{2}{c}{ Electrode without coating } & \multicolumn{3}{c}{ Electrode with coating } \\
\cline { 2 - 5 } & $\boldsymbol{i}_{\text {cor }, \mathbf{A} / \mathbf{m}^{2}}$ & $-\boldsymbol{E}_{\text {cor }, \mathbf{~ V}}$ & $\boldsymbol{i}_{\text {cor }, \mathbf{A} / \mathbf{m}^{2}}$ & $\boldsymbol{Z}, \boldsymbol{\%}$ & $-\boldsymbol{E}_{\text {cor }}, \mathbf{V}$ \\
\hline 0.25 & 0.29 & 0.47 & 0.10 & 65 & 0.47 \\
24 & 0.20 & 0.57 & 0.03 & 85 & 0.48 \\
48 & 0.25 & 0.61 & 0.028 & 89 & 0.47 \\
96 & 0.24 & 0.57 & 0.025 & 89 & 0.43 \\
120 & 0.25 & 0.54 & 0.04 & 84 & 0.50 \\
144 & 0.23 & 0.48 & 0.05 & 78 & 0.52 \\
168 & 0.24 & 0.42 & 0.10 & 58 & 0.50 \\
\hline
\end{tabular}

It follows from Table 3 that for 5 days the superhydrophobic coating of the III type protects the electrode with a value of $Z=84-89 \%$. On the sixth and seventh days, the $E_{\text {cor }}$ of the protected electrode becomes more negative than that of the unprotected one and the $Z$ value decreases to $58 \%$ on the 7th day. On a hydrophobic electrode, the cathodic process is inhibited and the anodic process is facilitated in comparison with an unprotected electrode (Figure 5).

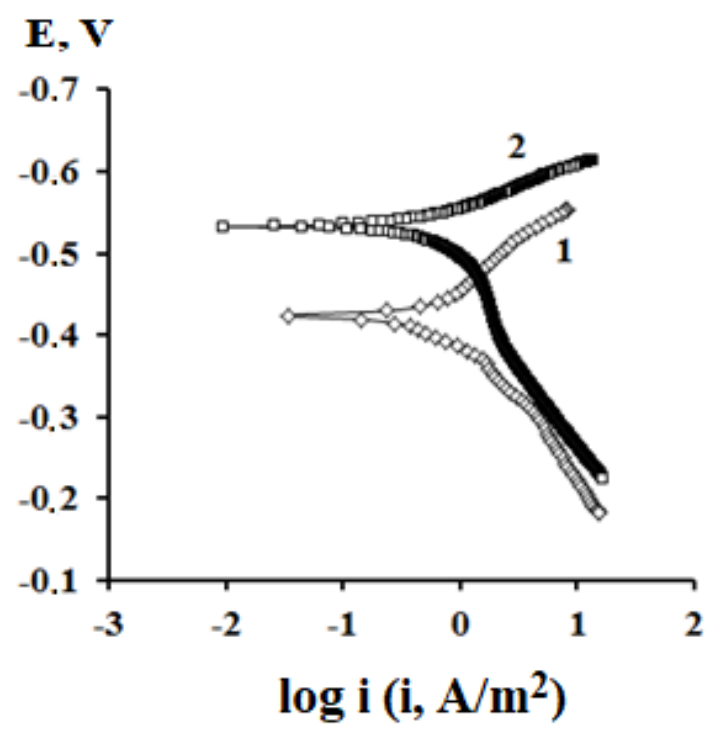

Figure 5. Polarization curves measured on a steel electrode without coating (1) and with type III superhydrophobic coating (2) after exposure to NACE medium $+400 \mathrm{mg} / \mathrm{L} \mathrm{H}_{2} \mathrm{~S}$ solution for $168 \mathrm{~h}$. 
Let us compare the protective effects of the superhydrophobic coating, calculated from the data of gravimetric tests and polarization measurements (Tables 2 and 3). According to 24 hour measurements, they practically coincide. The value of $Z$ calculated after 10 days of gravimetric tests of samples with the IV type of coating (65\%) turns out to be higher than on the 7th day of polarization measurements of samples with the III type of coverage (58\%). But this is understandable, since the latter are distinguished by the fact that they were not exposed to ultraviolet irradiation in the presence of ozone plasma, which contributed to the strengthening of the bond of fluorooxysilane molecules with the metal surface [11]. In this regard, the superhydrophobic properties of the coating of the III type are worse than those of the coating of the IV type.

We present the data in purely chloride media. According to [12], in the case of corrosion of carbon steel with superhydrophobic coatings of the I and II types in a $0.5 \mathrm{M} \mathrm{NaCl}$ solution, a steady state is achieved in 72 hours. In this case, the coefficient of inhibition of corrosion by the superhydrophobic coating $\gamma$ is $23 \pm 3$, which corresponds to the protective effect $Z=0.956 \pm 0.006$. This value is significantly higher than in environments with $\mathrm{H}_{2} \mathrm{~S}$.

The dynamics of the decrease in the protective properties of superhydrophobic coatings over time in environments containing hydrogen sulfide indicates that they cannot provide long-term effective protection, as at $100 \%$ humidity and in chloride solutions, which significantly more severely simulate atmospheric corrosion conditions in a maritime climate.

Thus, it can be stated that the investigated superhydrophobic coatings of the I, II, and IV types can be successfully used for long-term protection of carbon steel products under conditions of $100 \%$ humidity and neutral chloride environments.

The presence of $\mathrm{H}_{2} \mathrm{~S}$ and a more acidic environment than a neutral chloride solution determine the fragility of their protective effect. The use of ultraviolet irradiation in the presence of ozone plasma in the process of forming superhydrophobic coatings helps to increase the stability of the superhydrophobic state and improve their anticorrosive properties.

\section{Conclusion}

- Superhydrophobic coatings of I, II and IV types have high anticorrosive properties in conditions of $100 \%$ humidity and neutral chloride solution.

- The medium NACE with $\mathrm{pH}=3.6$ and the presence of $\mathrm{H}_{2} \mathrm{~S}(400 \mathrm{mg} / \mathrm{L})$ determines the fragility of the anticorrosive properties of the superhydrophobic coating.

- Superhydrophobic coatings, during the formation of which the treatment with ultraviolet irradiation in the presence of ozone plasma is used, exhibit a higher anticorrosive protection of carbon steel than without treatment.

- Determinations of the protective effectiveness of coatings by direct corrosion tests and on the basis of polarization curves are in satisfactory agreement with each other. 


\section{Acknowledgments}

This work was supported by the Russian Science Foundation, grant No. 18-16-00006.

The experimental results were obtained using the equipment of the Center for Collective Use of Scientific Equipment of TSU named after G.R. Derzhavin.

\section{References}

1. L.B. Boinovich, K.A. Emel'yanenko, A.G. Domantovsky and A.M. Emel'yanenko, Laser tailoring the surface chemistry and morphology for wear, scale and corrosion resistant superhydrophobic coatings, Langmuir, 2018, 34, no. 24, 7059-7066. doi: 10.1021/acs.langmuir.8b01317

2. D.A. Alpysbaeva, D.B. Vershok, A.M. Emel'yanenko, O.V. Batishchev, Yu.I. Kuznetsov and L.B. Boinovich, Superhydrophobization of low-carbon steel by surface coatings, Korroz.: Mater., Zashch. (Corrosion: materials, protection), 2013, no. 8, 42-47 (in Russian).

3. K.A. Emel'yanenko, N.A. Sanzharovsky, E.V. Chulkova, A.A. Ganne, A.M. Emel'yanenko and L.B. Boinovich, Superhydrophobic corrosion resistant coatings for copper via IR nanosecond laser processing, Mater. Res. Express, 2018, 5, no. 11, 115001. doi: $\underline{10.1088 / 2053-1591 / \text { aadc16 }}$

4. Z. Hua, J. Yang, T. Wang, G. Liu and G. Zhang, Transparent surface with reversibly switchable wettability between superhydrophobicity and superhydrophilicity, Langmuir, 2013, 29, no. 33, 10307-10312. doi: $10.1021 / 1 \mathrm{a} 402584 \mathrm{v}$

5. V.I. Vigdorovich, E.Yu. Shel, L.E. Tsygankova, N.V. Shel and A.A. Uryadnikov, The effect of the concentration of hydrogen ions on the kinetics of partial electrode reactions on steels with a superhydrophobic surface in acid chloride environments, Korroz.: Mater., Zashch. (Corrosion: materials, protection), 2017, no. 4, 1-11 (in Russian).

6. L.B. Boinovich, A.M. Emel'yanenko, A.D. Modestov, A.G. Domantovsky and K.A. Emel'yanenko, Not simply repel water: the diversified nature of corrosion protection by superhydrophobic coatings, Mendeleev Commun., 2017, 27, no. 3, 254256. doi: 10.1016/j.mencom.2017.05.012

7. J. Ou, M. Liu, W. Li, M. Xue and C. Li, Corrosion behavior of superhydrophobic surfaces of Ni alloys in NaCl solutions, Appl. Surf. Sci., 2012, 258, no. 10, 4724-4728. doi: 10.1016/j.apsusc.2012.01.066

8. V.I. Vigdorovich, L.E. Tsygankova, A.M. Emel'yanenko, M.N. Uryadnikova and E.Yu. Shel, The effect of superhydrophobic coating on the electrochemical behavior of carbon steel in chloride and hydrogen sulfide-chloride environments, Int. J. Corros. Scale Inhib., 2020, 9, no. 1, 171-181. doi: 10.17675/2305-6894-2020-10-1-10

9. V.I. Vigdorovich and S.A. Zakurnaev, Evaluation of the contributions of the polysulfide film and inhibitor in protecting steel against hydrogen sulfide corrosion, Korroz.: Mater., Zashch. (Corrosion: materials, protection), 2009, no. 2, 17-22 (in Russian). 
10. L.B. Boinovich and A.M. Emel'yanenko, The behaviour of fluoro- and hydrocarbon surfactants used for fabrication of superhydrophobic coatings at solid/water interface, Colloids Surf., A, 2015, 481, 167-175. doi: $\underline{10.1016 / j . c o l s u r f a .2015 .05 .003}$

11. L. Boinovich and A. Emel'yanenko, A wetting experiment as a tool to study the physicochemical processes accompanying the contract of hydrophobic and superhydrophobic materials with aqueous media, Adv. Colloid Interface Sci., 2012, 179, 133-141. doi: 10.1016/j.cis.2012.06.010

12. V.I. Vigdorovich, L.E. Tsygankova, A.A. Uryadnikov, N.V. Shel, L.G. Knyazeva, and E.D. Tanygina, The Effect of Nanocomposite Superhydrophobic Coating on Corrosion and Kinetics of Electrode Processes on Steel in $0.5 \mathrm{M} \mathrm{NaCl}$ Solution, Prot. Met. Phys. Chem. Surf., 2017, 53, no. 7, 1259-1264. doi: 10.1134/S2070205117070176 